\title{
Student experiences of virtual reality: A case study in learning special relativity
}

\author{
Dominic McGrath \\ Teaching and Educational Development Institute, The University of Queensland, St. Lucia, \\ Qld 4072, Australia \\ Margaret Wegener and Timothy J. Mclntyre \\ School of Mathematics and Physics, Faculty of Science, The University of Queensland, St. Lucia, \\ Qld 4072, Australia \\ Craig Savage and Michael Williamson \\ Physics Education Centre, The Australian National University, Canberra, Australian Capital Territory 0200, \\ Australia
}

(Received 28 October 2009; accepted 29 April 2010)

\begin{abstract}
A teaching package has been developed centered around a relativistic virtual reality. It introduces concepts of special relativity to students in a gamelike environment where users experience the effects of traveling at near light speeds. From this perspective, space and time are significantly different from that experienced in everyday life. We explore how students worked with this environment and how they used this experience in their study of special relativity. Students found the simulation to be a positive learning experience and described the subject area as being less abstract after its use. Students were more capable of correctly answering concept questions relating to special relativity, and a small but measurable improvement was observed in the final exam. (C) 2010 American Association of Physics Teachers.
\end{abstract}

[DOI: 10.1119/1.3431565]

\section{INTRODUCTION}

Abstract physics is concerned with the development and analysis of conceptual models of physics and explores the consequences of theory. The goals of courses on an abstract physics topic are to excite students about the subject area, provide a rationale for the relevant models, and develop abstract thinking abilities. However, many students undertake a surface learning approach ${ }^{1}$ and focus on manipulating formulas in place of developing an understanding of abstract principles. Although this approach might enable students to achieve satisfactory results in an assessment task, these students have learned little to support their future studies.

A common introduction to abstract physics is the study of special relativity, which is fundamentally and mathematically simple, popular, and provides access to an essentially different understanding of time and space. However, many students fail to develop an understanding of the fundamental concepts in special relativity even after advanced instruction. ${ }^{2}$ Understanding relativity requires one to accept that there is less that is absolute than was once believed and to accept a model of time and space that is strange and unfamiliar. ${ }^{3}$ Modifying everyday concepts of motion, time, and space to develop accurate constructs of the theory of special relativity is extraordinarily difficult. ${ }^{4,5}$

Mermin $^{3}$ and Scherr ${ }^{2}$ described courses that support students' learning and avoid common pitfalls. Ideas and recommendations for how and what special relativity should be taught in introductory courses have been discussed in Refs. 6-9.

Experiments that support the study of special relativity are limited because the effects of special relativity become significant only at near light speeds. Experiments have been developed to verify length contraction and time dilation by examining particles moving at near light speed. ${ }^{10-12}$ These experiments provide evidence to aid students' acceptance of special relativity, but they use a heavily guided approach to ensure the collection of appropriate data.

An alternative is the development of visualizations and simulations to aid the conceptual understanding of special relativity through imagery and virtual experiences. Gamow's Mr. Tompkins in Wonderland ${ }^{13}$ provides vivid descriptions of a relativistic world. More recently, computer-generated images and video that provide accurate imagery of motion at near light speed have been developed by Weiskopf et al. ${ }^{14}$ Taylor $^{15}$ described an educational implementation of several special relativity simulations, including a wire-frame threedimensional simulation identifying visual effects of special relativity, and a model of a two-dimensional world featuring clocks. Taylor's two-dimensional simulation features associated space-time diagrams, enabling exploration and connection between multiple representations of space and time. Belloni et al. ${ }^{16}$ developed simulations based on Scherr's activities. Carr et al. ${ }^{17}$ developed serious games employing the effects of special relativity. Recent technological advances have enabled the development of more sophisticated simulations. ${ }^{14}$ An example is the virtual reality simulation developed by Savage et al. ${ }^{18}$ which models the visual, spatial, and temporal effects of special relativity. This software, known as REAL TIME RELATIVITY, is implemented on a personal computer with a programmable graphics card and provides an opportunity for new approaches to learning special relativity.

This paper describes the implementation and evaluation of REAL TIME RELATIVITY and associated learning activities as performed over four semesters. The study builds on an earlier smaller scale study $^{18}$ by accessing an expanded student cohort from two research-intensive institutions. The Australian National University (ANU) attracts students from around the country, while students of The University of Queensland (UQ) tend to originate from the state of 


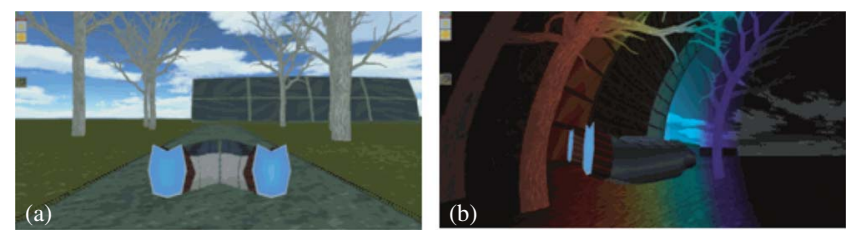

Fig. 1. Sample screen shots from the REAL TIME RELATIVITY software. (a) Cityscape scenario at zero relative velocity. (b) Moving through the cityscape at 0.866 times the speed of light.

Queensland and from Asia. At these universities, REAL TIME RELATIVITY has been incorporated in first year physics courses where special relativity and quantum mechanics serve as an introduction to abstract physics. An important aspect of this incorporation is enabling students to recognize the value of abstract physics and to develop a desire and a basis for further physics study.

\section{IMPLEMENTATION}

The study began by using the existing version of REAL TIME RELATIVITY as the basis of a laboratory activity at both institutions. Laboratory classes were chosen as they are the traditional setting for interaction and experimentation in physics courses. Laboratory sessions enable groups of students to actively and collaboratively explore phenomena and to develop, test, and utilize theory. Also, the computers required to run the software were most readily available in the teaching laboratories.

The software simulates the visual effects that become apparent when traveling at near light speed. Users participate in a gamelike world, with control over their motion and direction of view. Scenarios include environments consisting of clocks, planets, spheres, planes, and prisms. Some effects can be disabled to focus on particular concepts and to allow the user to become accustomed to navigation controls and the scenarios; otherwise, the visual experience of the user is extremely accurate. The previous study ${ }^{18}$ had identified some difficulties with the navigational aspects of the software, which were addressed during the early stages of this implementation. Updated scenarios were also made available to the students. Sample screen images are shown in Fig. 1.

REAL TIME RELATIVITY was used by students in 3-h laboratory sessions. These classes contained small groups of students working in pairs. At ANU, students completed the experiments in parallel with a lecture series on special relativity. At UQ, there was a shorter set of lectures, and the laboratory was part of a series of experiments that students completed on a rotating basis throughout the semester. Hence, students attempted the experiments before, during, or after the lecture and tutorial series on special relativity. Students at each institution were expected to prepare for the laboratory practical by reviewing materials in textbooks and online in video and text formats, and completing a series of preliminary questions. On arrival in the laboratory their responses were checked by tutors, who were also available to provide guidance throughout the session.

Throughout this study, learning activities and resources were varied to examine possible uses of REAL TIME RELATIVITY, while maintaining a peer-supported active learning approach. The final version of the laboratory session at UQ is as follows. Students were initially encouraged to familiarize themselves with the interface and environment of REAL TIME
RELATIVITY by exploring the various scenarios and controls available. Students were then required to use REAL TIME RELATIVITY to connect phenomena of special relativity and the finite speed of light to their observations. For example, students had to determine how length contraction appears in REAL TIME RELATIVITY and disentangle it from other effects, such as the light propagation delay. They were then asked to use words and drawings to explain their observations using relativity theory. In this stage, students' concepts were challenged and refined by comparison with observations and experiments in REAL TIME RELATIVITY. Finally, students had to apply their understanding to develop and carry out an experiment to quantitatively verify time dilation. Students used a series of clocks within a REAL TIME RELATIVITY environment and recorded times while traveling at various speeds. Effects such as length contraction and optical aberration had to be considered in the design of their experiment. During these activities, students worked collaboratively. Students were required to keep a log book record of their work featuring annotated diagrams to demonstrate the connections they made between the theory and the visual effects. The log books were collected at the end of the session and compared to the students' ability to demonstrate their understanding of the topics as evidenced by surveys, quizzes, and exams.

The laboratories conducted in Semester II 2009 at UQ included eight problems. Each included a short description and references. The first five asked students to use text and diagrams to describe how particular effects of special relativity are represented in the software. In order, students examined light delay, time dilation, optical aberration, length contraction, and the Doppler effect. These problems challenged students to develop and question their mental models through having to observe, test, and provide evidence for these effects. Problem six asked students to consider a different reference frame to that of the ship provided by REAL TIME RELATIVITY. They were asked to describe the appearance of the ship itself if it was moving at $0.866 c$. The final two problems required a quantitative confirmation of light delay. Students had to design a process for collecting temporal data that accounted for other effects, such as optical aberration. After developing a design, students collected data and confirmed the time dilation formula.

Data about the student learning experiences was collected in 2008 and 2009, in Semester II at ANU and in Semesters I and II at UQ. (Preliminary results for 2008 are discussed in Ref. 19.) The study involved more than 300 students (out of the 420 students in the relevant courses). Students participating in this research were from a variety of majors, mainly engineering and science. For some students, the course was required within an engineering program. The laboratory activities at UQ were offered on a randomly assigned rotational basis so that not all students completed all laboratory activities.

Data collection targeted user confidence, self-efficacy, and attitudes toward physics, special relativity, and technology, as well as level of performance on assessment tasks and how students were learning. A variety of methods were used. Before and after completing their laboratory session, participants responded to closed and open survey questions, completed confidence $\operatorname{logs},{ }^{20}$ and were administered conceptbased multiple choice tests. To avoid repetition effects and bias in the concept tests, sets of questions randomly assigned as pre- and postlaboratory were used. Survey questions probed beliefs about space and time that are challenged by 
Table I. Means and standard deviations for prelaboratory survey results. Responses scored as 1 - strongly disagree, 2-disagree, 3-neutral, 4-agree, and 5-strongly agree. Sample sizes were 31 at ANU and 187 at UQ. A $95 \%$ confidence interval for the error in the means is less than 0.3 for ANU and 0.2 for UQ.

\begin{tabular}{lcc}
\hline \hline & ANU & UQ \\
\hline Special relativity is more abstract than other areas of & $3.6 \pm 1.1$ & $3.9 \pm 0.9$ \\
physics. & $3.2 \pm 0.8$ & $2.9 \pm 0.9$ \\
I have a good understanding of special relativity. & $2.6 \pm 0.8$ & $3.1 \pm 1.0$ \\
I can use the formulae for special relativity but do not & & $3.9 \pm 0.9$ \\
understand why they work. & $4.0 \pm 0.8$ & $4.0 \pm 0.8$ \\
I enjoy trying new things on a computer. & $4.1 \pm 0.8$ & \\
I find simulations are an effective way to learn. & & \\
\hline \hline
\end{tabular}

the study of special relativity. Randomly selected student groups were observed working in the laboratory and informally interviewed when clarification was required. Focus groups formed at the end of the semester from five to ten student volunteers provided, at least for this sample of students, an in-depth view of the role of the laboratory activity in their learning, as well as some clarification of the survey findings. Students were administered an online survey at the completion of the series of special relativity lectures and tutorials when approximately half the class had used REAL TIME RELATIVITY. Randomly selected student laboratory work was reviewed. Performance on the final examination was compared for students who had been assigned to the REAL TIME RELATIVITY laboratory and for those who had not.

The REAL TIME RELATIVITY software, the associated teaching package, and surveys can be downloaded from Ref. 21 or Ref. 22.

\section{RESULTS}

\section{A. Student attitudes}

A prelaboratory survey probed students' attitudes and views of themselves in relation to their ability to understand special relativity and their interests in using simulations as a learning tool. The results are shown in Table I. As expected, students indicated that they perceived special relativity as being a more abstract area of physics based on their previous experiences. There was a fairly neutral response to students' beliefs about their understanding of special relativity. UQ students rated slightly lower in this question, which may have been influenced by the rotating nature of the laboratory experiments. UQ students were also more likely to believe that they could mechanically apply the equations of special relativity without having a good understanding of the associated physics.

Students were queried about their interests in using computers for learning and, in particular, their desire to use computer simulations to illustrate concepts in physics. The positive outcomes indicated that the surveyed students enjoyed using simulations. There is a substantial literature demonstrating the benefits of simulations for promoting student learning and engagement. ${ }^{23}$

A postlaboratory survey was completed by students immediately after the REAL TIME RELATIVITY laboratory. The results are shown in Table II. Students left the laboratory interested in special relativity and with a desire to learn more about the topic. This desire is consistent with earlier findings. ${ }^{18}$ Students also indicated that the simulations were more fun than other experiments, which might relate to the gamelike nature of the simulations. ANU students indicated an interest in making further use of the software on their own time; this motivation was not as strong among the UQ students. The postlaboratory survey included a final response section for open comments. Although this section was utilized by only about $15 \%$ of the respondents, a significant fraction identified the activity as either fun or helpful for understanding and learning.

Students were asked to complete confidence logs immediately before and after the laboratory activities. Students rated their own confidence with regard to various aspects of special relativity on a scale ranging from no confidence to very confident. $^{20}$ Students' confidence ratings immediately prior

Table II. Means and standard deviations for postactivity survey results. Responses scored as in Table I. Sample sizes were 31 at ANU and 175 at UQ. The 95\% confidence interval for the error in the means is less than 0.3 for ANU values and 0.2 for UQ values.

\begin{tabular}{|c|c|c|}
\hline & ANU & UQ \\
\hline I would like to learn more about special relativity. & $4.3 \pm 0.7$ & $3.7 \pm 1.0$ \\
\hline $\begin{array}{l}\text { I would be interested in using the REAL TIME RELATIVITY } \\
\text { software in my own time. }\end{array}$ & $3.6 \pm 0.7$ & $3.0 \pm 1.1$ \\
\hline $\begin{array}{l}\text { In other experiments it was easier to connect the theory } \\
\text { to what I observed. }\end{array}$ & $2.9 \pm 1.1$ & $3.1 \pm 1.1$ \\
\hline $\begin{array}{l}\text { Using a relativity simulation is more fun than the other } \\
\text { experiments. }\end{array}$ & $3.7 \pm 0.8$ & $3.5 \pm 1.0$ \\
\hline I learnt more from this experiment than most others. & $3.3 \pm 0.9$ & $3.4 \pm 1.0$ \\
\hline I would like to use more simulations in my studies. & $3.7 \pm 0.7$ & $3.6 \pm 1.0$ \\
\hline I found this to be an interesting experiment. & $4.0 \pm 0.6$ & $3.9 \pm 0.8$ \\
\hline
\end{tabular}


Table III. Students' self-assessment of confidence level for specific tasks. The numbers are the probability of no improvement in confidence (measured by paired $t$-test) after completing the REAL TIME RELATIVITY activities. Sample sizes were $N=148$ at ANU and 30 at UQ.

\begin{tabular}{lrr}
\hline \hline Task & $\begin{array}{r}\text { ANU } \\
(\%)\end{array}$ & $\begin{array}{r}\text { UQ } \\
(\%)\end{array}$ \\
\hline $\begin{array}{l}\text { Explain the theory of special relativity to someone who } \\
\text { isn't studying physics } \\
\begin{array}{l}\text { Apply aspects of the theory of special relativity to solve } \\
\text { problems }\end{array}\end{array}$ & $<0.1$ & $<0.1$ \\
$\begin{array}{l}\text { Calculate the length contraction of a moving object } \\
\text { given a relative velocity }\end{array}$ & 0.9 & 3 \\
$\begin{array}{l}\text { Predict the change in color of an object moving at near } \\
\text { light speed }\end{array}$ & 0.1 & $<0.1$ \\
$\begin{array}{l}\text { Describe the observed changes in shape of an object } \\
\text { moving at high speed }\end{array}$ & $<0.1$ & $<0.1$ \\
\hline \hline
\end{tabular}

to and immediately after completion of the activities were compared (using a paired $t$-test to test the hypothesis of no improvement in confidence for each aspect). The results, shown in Table III, indicate that the confidence of students at each university increased in all the aspects of special relativity that were measured. Although indications of improved student confidence do not directly imply improvements in learning, they do indicate a change in students' perception, understanding, and affective connection to a topic, which can lead to improvements in learning. A notable outlier in confidence development for UQ students was length contraction, an effect that is traditionally demonstrated in one or two dimensions and is quantified by a simple mathematical expression. REAL TIME RELATIVITY demonstrates length contraction in three dimensions, with the added complexities of other visual effects, challenging students to make complex connections between length contraction and other effects of special relativity.

One goal of the study was to provide a learning environment that presents special relativity in a less abstract way. Studies indicate that students perceive a topic area as abstract when it is presented as a mathematical model without meaning, application or connection to the physical world. REAL TIME RELATIVITY provides a visualization that complements the mathematical formalism of special relativity. The connection of special relativity to the human scale is made explicit as students experience relativistic effects at speeds approaching that of light. Through these experiences students identify special relativity as less abstract. The online survey provides evidence of this change (see Fig. 2). Students who used REAL

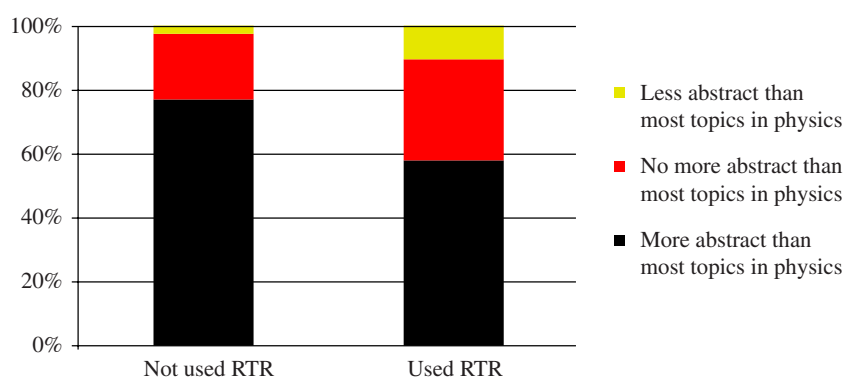

Fig. 2. Results from the online survey of students on the abstractness of special relativity (ANU Semester II, 2008, $N=54$ ).
TIME RELATIVITY were less likely than those who hadn't to identify special relativity as more abstract than most topics in physics; some even perceived it as less abstract than most physics topics.

\section{B. Learning outcomes}

Although students' perceptions of their level of learning are important in judging the effectiveness of a teaching innovation, it is at least equally important to examine the learning outcomes based on independent testing. Students were presented with a short concept test before and after the REAL TIME RELATIVITY activities. The test consisted of a set of belief statements requiring students to express their level of agreement or disagreement. The results of selected questions from the test are shown in Fig. 3.

Performance on these concept questions generally improved across a broad range of topics, showing an overall trend toward a deeper understanding of special relativity. In particular, questions concerning time dilation and simultaneity showed significant increased understanding based on answers to pre- and postlaboratory questions $(t$-test $p<0.05)$. The improvement in time dilation was expected because time dilation is a specific focus in the laboratory activities. The improvements in understanding of simultaneity were unexpected as simultaneity was not an explicit focus of the activities. Not all concept questions asked resulted in useful outcomes. In some cases, concepts were well understood prior to the laboratory activity, resulting in only an insignificant change in understanding. (These have not been included here.) In other cases, responses on less well understood topics showed no significant improvement. For example, the question on the existence of the correct order of events is shown in Fig. 3.

Focus group and open-ended survey responses indicated that students learned in a visual manner, reflecting the visual nature of REAL TIME RELATIVITY. Students reported gaining "an ability to visualize relativistic effects which make it easy to apply theory," that "it was much easier to learn the concepts (ALL concepts) of relativity when it is seen visually," and "it helped a lot with understanding because you could visualize something, that you have no experience of visualizing in real life." These responses were reinforced when students explained how they approached problems in terms of visual models and examples from their experiences in REAL TIME RELATIVITY. "When we did the lab in here, it reinforced all the ideas and also made it clearer-Oh this is what happens you can visualize it 'cause it is not something you can see every day."

Students at UQ in Semester II, 2008, were randomly assigned either to a group that completed the REAL TIME RELATIVITY experiment $(N=134)$ or to a control group who did not $(N=51)$ due to the rotating nature of the laboratory sessions. Responses on the final exam were compared for these two groups. The final exam question on relativity was prepared and marked externally to this study and was based on material presented in three 1-h lectures and one tutorial session. Students were expected to conduct at least $6 \mathrm{~h}$ of further independent study. It was found that students who completed the simulations performed better on the special relativity section. Although the effect size was small (Cohen's $d=0.33$ ) ${ }^{24}$ the effect was statistically significant (probability of random occurrence $p<0.05$ by the unpaired $t$-test). A review of the exam papers did not reveal any significant 


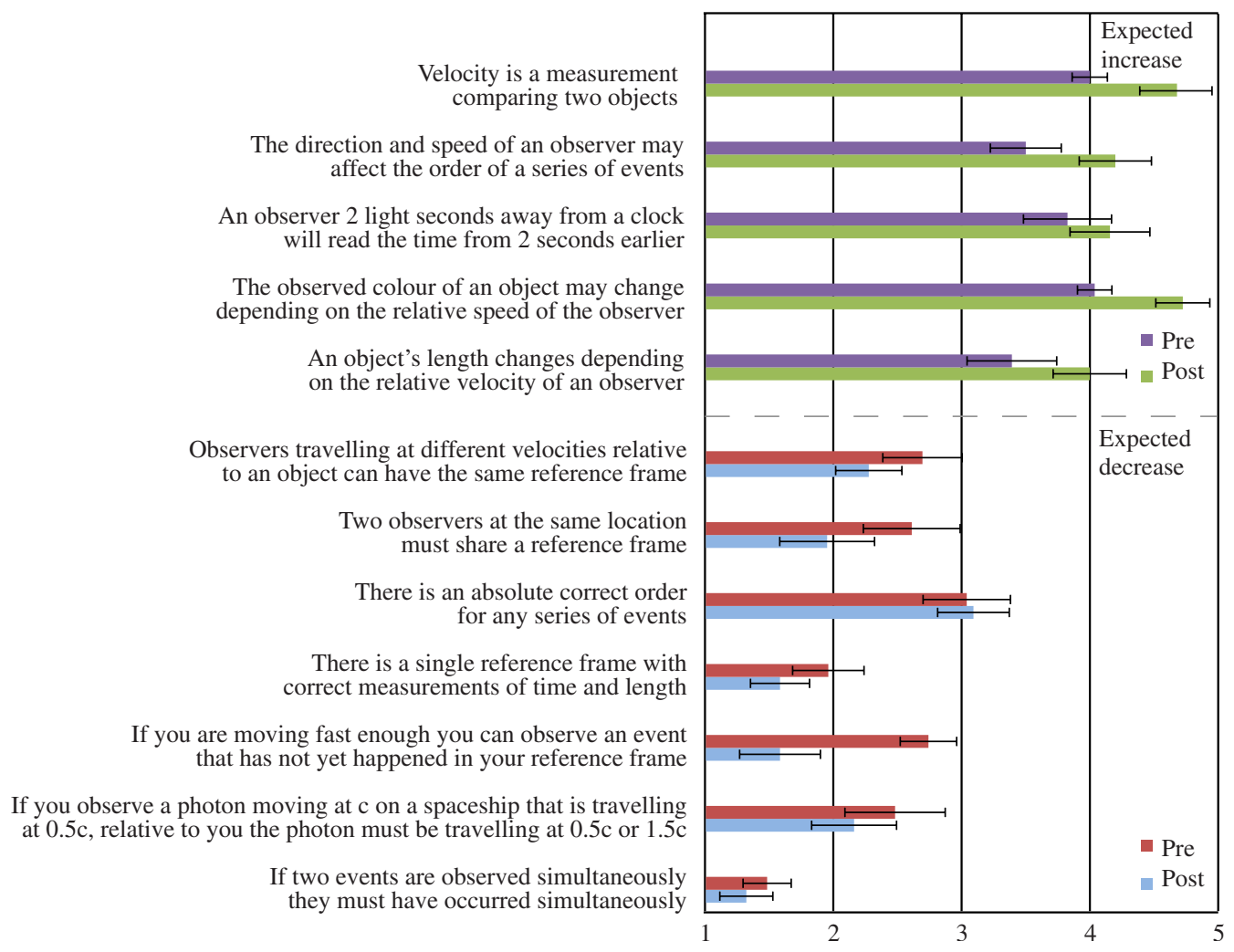

Fig. 3. Average of student responses to statements before and after the REAL TIME RELATIVITY activities. The questions are divided into those where the statements are true (upper half) and those that are false (lower half). Responses scored as in Table I. Black lines show the standard deviation of the mean (UQ Semester I, 2009, $N=28)$.

difference in the approach between these groups. Students who completed the REAL TIME RELATIVITY activities were also found to have performed better on a question relating to quantum mechanics but no correlations were found with other topics.

A similar analysis of exam results for students at UQ in Semester II, 2009, was performed. The trend was similarREAL TIME RELATIVITY users performed better on the exam questions relating to relativity and quantum physics and did not perform better on any other topics. However, in this case, with $p=0.13$ by an unpaired $t$-test, there is a $13 \%$ chance that the better performance on relativity questions was due to random variation, so the evidence is not conclusive. In the future, all students will be required to complete the REAL TIME RELATIVITY activities so it will not be possible to make further comparisons.

\section{Nature of learning}

Responses to postlaboratory open questions about what and how students learned were classified using categories derived from the student responses. The classifications were reviewed and discussed by two members of the research team until consensus was reached. Students identified the most interesting aspect of the REAL TIME RELATIVITY activities to be exploring an individual effect or a selected group of effects of special relativity (68\% ANU students, $80 \%$ UQ students). Students learned about these effects through "doing" (20\% ANU, 45\% UQ), "observing," including seeing or watching (50\% ANU, 36\% UQ), tutor-led discussions (10\% ANU, 10\% UQ), and reading supplementary material (20\% ANU, 7\% UQ), as shown in Fig. 4, demonstrating a predominantly active approach to learning. At least $70 \%$ of student responses at both institutions could be classified as typical descriptions of students undertaking experimental work. Thus, virtual reality can be seen to be providing learning opportunities equivalent to other laboratory activities. Students also identified value in the active, student-led approach. For example: "The section where you had to design an experiment about time dilation was useful ...you had to design and you weren't just told what to do." Students also explicitly identified discussions with their laboratory partners as a way in which they learned.

Observations of student interactions during the laboratory sessions yielded further insights into the learning process. Students developed deeper understanding by negotiating the theoretical justifications for their observations and challenging inaccurate conceptions through debate and experimentation. Most students' initial experience of increasing speed involved moments of "Hang on, why are we going backwards?," as aberration had a greater effect on perspective than motion. By a process of testing travel at constant speed and increasing and decreasing speeds, students developed an understanding of aberration. Students then had to explicitly connect their experiences to theory by describing how these effects matched the theory. This matching of observation to labeled effects involved discussion within student groups, testing of concepts, and guidance from laboratory tutors. Students commented that "initially it was overwhelming — but it gets in your head by the end." As students moved through various tasks in the laboratory activities, this progression through initial confusion to understanding, refinement, and connection to theory was repeated. The Doppler effect, 


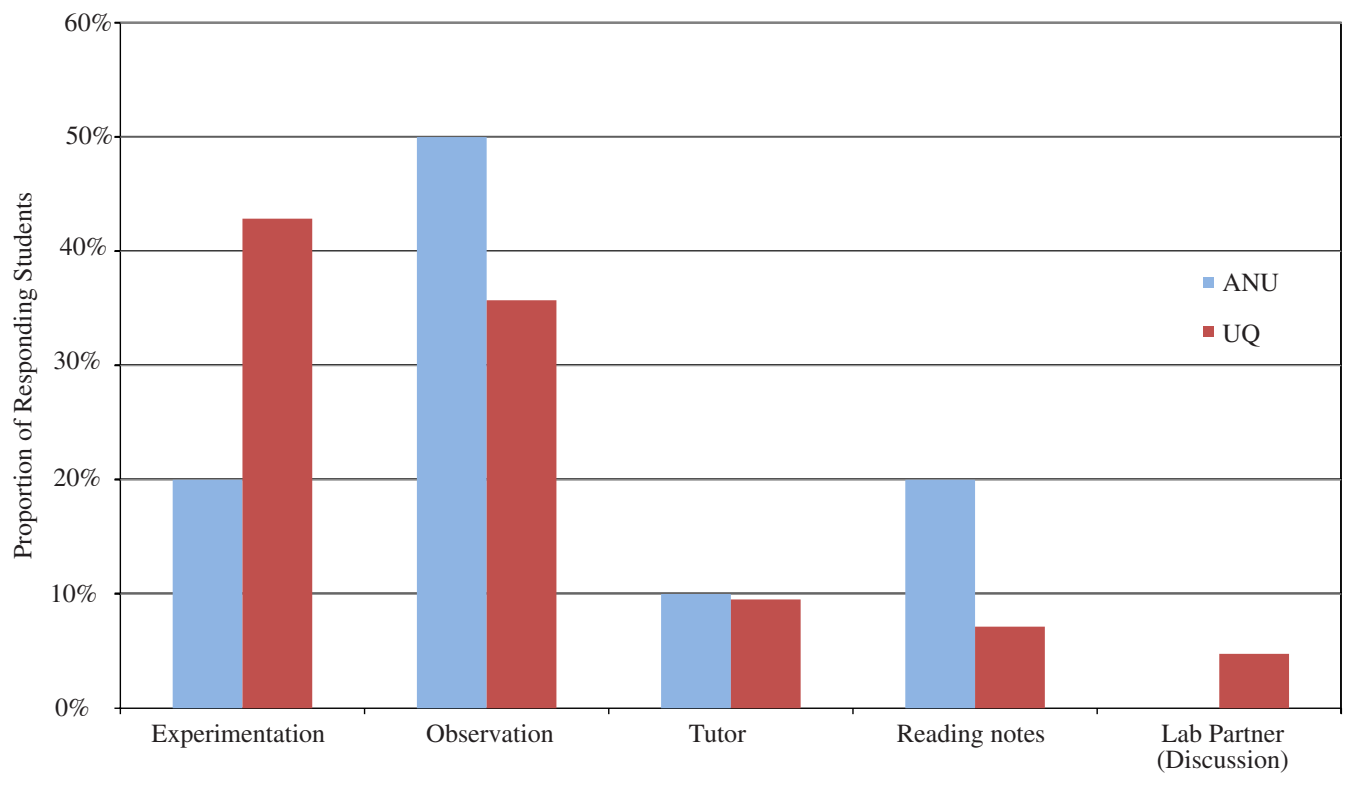

Fig. 4. Classifications of students' responses to open questions about how they learned through the REAL TIME RELATIVITY activities (ANU and UQ, $N=52$ ).

which was initially disabled for the experiment, inspired comments such as "It's pretty but I don't know what it is" when turned on. With the support of written material, peers, and tutors, these conversations quickly moved to more mature analysis. For example, student 1: "Why is there a section losing color?" student 2: "It's because infrared and ultraviolet and other wavelengths are outside the visible spectrum." These conversations consistently incorporated appropriate terminology and demonstrated the development of appropriate models.

Students who used REAL TIME RELATIVITY after lectures or tutorials on special relativity reported benefits from their activity. These students identified value in the concrete experience of virtual reality connecting to theory: "The lecturer was explaining aberration with the rain analogy, which I kind of understood but when I used REAL TIME RELATIVITY I went OK so that is what it is." ..."I was able to refine my knowledge about some phenomenon and also finally start assigning the right names to things" ... "It helps show that while special relativity may seem counterintuitive it is because our intuition was not developed traveling at near the speed of light." For some students, their experiences challenged misconceptions they brought to the laboratory. "I thought things would look bigger because you get smaller" indicated a misconception regarding reference frames that the student then redeveloped with the support of the simulation, learning resources, and their peers.

Students who used REAL TIME RELATIVITY before their series of lectures and tutorials also identified some benefits. For example, "I have a friend who did it [the experiment] about halfway through special relativity lectures and I was talking to him about it at lunch today, and [he] went, 'Ah, now that you mention it,' because when he did this experiment, he suddenly understood - and that makes sense because I can pinpoint the time we were sitting in a row and would often be a little confused as a group of friends and think: It is this, but not quite be sure, and then halfway through he suddenly became more confident .... and now we know he did the experiment, so that makes sense."

\section{CONCLUSIONS}

REAL TIME RELATIVITY enables students to encounter phenomena that are outside human experience. The simulation along with an accompanying instructional package has been successfully implemented and studied in an undergraduate laboratory setting at two tertiary institutions. Data were collected through pre- and postlaboratory surveys and concept tests, focus groups, observation of student interactions during laboratory sessions, and through the analysis of exam papers completed by the students.

The study showed that students were able to develop visual models of the effects of special relativity through the use of the simulation. This model aided their understanding and enabled the students to see the topic as less abstract. Students enjoyed the learning experience and became more confident in their understanding of the topic. Postlaboratory evaluations showed that not only did students perceive that they had an improved understanding, they were also able to perform better in concept tests on special relativity. An analysis of exam scripts showed that completing the experiment sometimes aided in answering an exam question on relativity based on the lecture component of the course.

In the implementation of this virtual reality activity at two universities, there were some variations in the student types and in the presentation of other relevant learning activities. The fact that the package was robust enough to yield positive responses at each institution with different staff teams gives confidence that the REAL TIME RELATIVITY simulation and teaching package will perform favorably in other implementations.

The outcomes of this work show that virtual reality can aid in changing beliefs about reality and can assist in making traditionally difficult abstract topics more accessible. We are currently investigating other topics where a visual approach might aid in student learning and have begun work on a simulation of concepts in quantum mechanics. ${ }^{25}$ 


\section{ACKNOWLEDGMENTS}

Support for this study was provided by The Australian Learning and Teaching Council, an initiative of the Australian Government Department of Education, Science, and Training. The views expressed in this paper do not necessarily reflect the views of The Australian Learning and Teaching Council. The REAL TIME RELATIVITY simulator was originally developed at ANU by Lachlan McCalman, Anthony Searle, and Craig Savage.

${ }^{1}$ F. Marton and R. Saljo, "On qualitative differences in learning: I. Outcome and process," Br. J. Educ. Psychol. 46 (1), 4-11 (1976).

${ }^{2}$ R. E. Scherr, "An investigation of student understanding of basic concepts in special relativity," Ph.D. thesis, University of Washington, 2001.

${ }^{3}$ N. D. Mermin, It's About Time: Understanding Einstein's Relativity (Princeton U. P., Princeton, NJ, 2005).

${ }^{4}$ R. E. Scherr, P. S. Shaffer, and S. Vokos, "Student understanding of time in special relativity: Simultaneity and reference frames," Am. J. Phys. 69, S24-S35 (2001).

${ }^{5}$ R. E. Scherr, P. S. Shaffer, and S. Vokos, "The challenge of changing deeply held student beliefs about the relativity of simultaneity," Am. J. Phys. 70, 1238-1248 (2002).

${ }^{6}$ A. J. Mallinckrodt, "Relativity theory versus the Lorentz transformations," Am. J. Phys. 61, 760-760 (1993).

${ }^{7}$ N. D. Mermin, "Lapses in relativistic pedagogy," Am. J. Phys. 62, 11 (1994)

${ }^{8}$ M. S. Greenwood, "Relativistic addition of velocities using Lorentz contraction and time dilation," Am. J. Phys. 50, 1156-1157 (1982).

${ }^{9}$ A. Gjurchinovski, "Relativistic addition of parallel velocities from Lorentz contraction and time dilation," Am. J. Phys. 74, 838-839 (2006).

${ }^{10}$ T. Coan, T. Liu, and J. Ye, "A compact apparatus for muon lifetime measurement and time dilation demonstration in the undergraduate laboratory," Am. J. Phys. 74, 161-164 (2006).

${ }^{11}$ N. Easwar and D. A. MacIntire, "Study of the effect of relativistic time dilation on cosmic ray muon flux-An undergraduate modern physics experiment," Am. J. Phys. 59, 589-592 (1991).
${ }^{12}$ M. Lund and U. I. Uggerhoj, "Experimental special relativity with a meter stick and a clock," Am. J. Phys. 77, 757-761 (2009).

${ }^{13}$ G. Gamow, Mr. Tompkins in Paperback (Cambridge U. P., Cambridge, 1965).

${ }^{14}$ D. Weiskopf et al., "Explanatory and illustrative visualization of special and general relativity," IEEE Trans. Vis. Comput. Graph. 12 (4), 522534 (2006).

${ }^{15}$ E. F. Taylor, "Space-time software: Computer graphics utilities in special relativity," Am. J. Phys. 57, 508-514 (1989).

${ }^{16}$ M. Belloni, W. Christian, and M. H. Darcy, "Teaching special relativity using physlets," Phys. Teach. 42, 284-290 (2004).

${ }^{17}$ D. Carr, T. Bossomaier, and K. Lodge, "Designing a computer game to teach Einstein's theory of relativity," in CGIV '07: Proceedings of the Computer Graphics, Imaging and Visualisation, edited by E. Banissi, M. Sarfraz, and N. Dejdumrong (IEEE Computer Society, Washington, D.C., 2007), pp. 109-114.

${ }^{18}$ C. Savage, A. Searle, and L. McCalman, "Real time relativity: Exploratory learning of special relativity," Am. J. Phys. 75, 791-798 (2007).

${ }^{19}$ D. McGrath, C. Savage, M. Williamson, M. Wegener, and T. McIntyre, "Teaching special relativity using virtual reality," in Proceedings of the UniServe Science Symposium on Visualisation and Concept Development (Uniserve Science, The University of Sydney, Sydney, NSW, Australia, 2008), pp. 67-73.

${ }^{20}$ S. W. Draper, M. I. Brown, F. P. Henderson, and E. McAteer, "Integrative evaluation: An emerging role for classroom studies of CAL," Comput. Educ. 26 (1-3), 17-32 (1996).

${ }^{21}$ See $\langle$ www.anu.edu.au/Physics/vrproject//.

${ }^{22}$ See supplementary material at http://dx.doi.org/10.1119/1.3431565 for access to the associated teaching package and surveys.

${ }^{23}$ C. E. Wieman and K. K. Perkins, "A powerful tool for teaching science," Nat. Phys. 2, 290-292 (2006).

${ }^{24}$ Cohen's $d$ is a dimensionless measure of the size of a difference between two groups, defined to be the difference in means for each group divided by the standard deviation.

${ }^{25}$ C. Savage, D. McGrath, T. McIntyre, M. Wegener, and M. Williamson, "Teaching physics using virtual reality," in Proceedings of the International Conference on Physics Education, Bangkok, Thailand, 2009, edited by P. Wattanakasiwich (American Institute of Physics, College Park, MD, 2010).

\section{ONLINE COLOR FIGURES AND AUXILIARY MATERIAL}

AJP uses author-provided color figures for its online version (figures will still be black and white in the print version). Figure captions and references to the figures in the text must be appropriate for both color and black and white versions. There is no extra cost for online color figures.

In addition AJP utilizes the Electronic Physics Auxiliary Publication Service (EPAPS) maintained by the American Institute of Physics (AIP). This low-cost electronic depository contains material supplemental to papers published through AIP. Appropriate materials include digital multimedia (such as audio, movie, computer animations, 3D figures), computer program listings, additional figures, and large tables of data.

More information on both these options can be found at www.kzoo.edu/ajp/. 